\title{
Spectral-Temporal Classification Using Vegetation Phenology
}

\author{
ROBERT M. HARALICK, SENIOR MEMBER, IEEE, CHRISTINE A. HLAVKA, \\ RYUZO YOKOYAMA, MEMBER, IEEE, AND S. M. CARLYLE
}

\begin{abstract}
In this paper we describe a multitemporal classification procedure for crops in Landsat scenes. The method involves the creation of crop signatures which characterize multispectral observations as functions of phenological growth states. The phenological signature models spectral reflectance explicitly as a function of crop maturity rather than a function of date. This means that instead of stacking spectral vectors of one observation on another, as is usually done for multitemporal data, for each possible crop category a correspondence of time to growth state is established which minimizes the smallest difference between the given multispectral multitemporal vector and the category mean vector indexed by growth state. The results of applying it to winter wheat show that the method is capable of discrimination with about the same degree of accuracy as more traditional multitemporal classifiers. It shows some potential to label degree of maturity of the crop without crop condition information in the training set.
\end{abstract}

\section{INTRODUCTION}

$\mathrm{T}$ HE USUAL MODEL for classification of remotely sensed data relies on simple Gaussian statistical structure. For example, to discriminate a subclass of corn from a subclass of wheat we assume that data vectors (received energy as a function of band measured for a set of observation times) coming from each corn subclass are distributed according to one peaked (unimodal) probability distribution and data vectors coming from each wheat subclass are distributed according to another peaked probability distribution. This implicitly assumes that 1) the phenological growth stage for each vegetation subclass is the same for all observations made at a single time and 2) a multitemporal observation will have no missing data. For example, see Fu, Landgrebe, and Phillips [3] or papers in the Purdue Symposia on Machine Processing of Remotely Sensed Data. In regard to 1), however, it is well known that even in a geomorphologically homogeneous area, the phenological growth states are not the same, due to differences in planting time, soil types, and weather conditions. This results in probability distributions of crop reflectances which have large variances and which are sometimes not unimodal. Evidence for this is empirical: in an experiment with Landsat imagery taken on 5 dates in 1974 over the LACIE

Manuscript received October 3, 1979; revised December 12, 1979.

R. M. Haralick is with the Department of Electrical Engineering and the Department of Computer Science, Virginia Polytechnic Institute and State University, Blacksburg, VA 24062.

C. A. Hlavka was with the Center for Research, Inc., University of Kansas, Lawrence, KS 66044.

R. Yokoyama is with the Department of Electronics, Iwate University, Morioka Iwate 020, Japan.

S. M. Carlyle is with the Computer Science Department, University of Kansas, Lawrence, KS 66044. intensive test site in Morton County, KA, we determined that the standard deviation of wheat on a single band and date was 2.88 and on a single band conditioned by growth state and then averaged overall growth states was 1.42 . This data adds support to the assumption that the variation of phenological growth states increases the variance of the usual probability distribution (which is not conditioned by growth state) by many times. These larger variances can cause low-classification accuracy. In regard to 2), clouds are notorious creators of missing data.

A solution to the problems of missing data and low-classification accuracy can be achieved by relating spectral signature to crop phenology. Wheeler, Misra, and Holmes [6] and Misra and Wheeler [5] discuss some empirical relationships between vegetation phenology and multispectral reflectance. Kauth and Thomas [4] discuss one graphic approach to relating spectral signatures to crop phenology.

In this paper we begin from a Bayesian point of view. We assume that in most cases the probability of having a crop category given the observed multitemporal multispectral reflectances is zero except for one category. Therefore, Bayesian classification is done by eliminating inconsistent category choices. We work from the spectral reflectance for each category to the possible phenological growth states the category can have which are consistent with the observed spectral reflectance and crop calendar practices of the region. In this manner we determine a quickly implementable table lookup decision rule which uses crop phenology. This method explicitly assumes that it is meaningful for a category in a particular growth state to have a signature. It assumes no change in category over the period of observation.

To illustrate the meaning of this, consider a 2-band simple first-order example. Suppose a 2-band spectral observation $\left(\alpha_{1}\left(t_{1}\right), \alpha_{2}\left(t_{1}\right)\right)$ is taken at time $t_{1}$, using wavelength $\left(\lambda_{1}, \lambda_{2}\right)$. This can be classified by determining for each category all those phenological growth states of vegetation of category $c$ which can yield spectral return $\alpha_{1}\left(t_{1}\right)$ at wavelength $\lambda_{1}$ and spectral return $\alpha_{2}\left(t_{2}\right)$ at wavelength $\lambda_{2}$. If there is not a phenological growth stage of category $c$ which yields spectral returns $\alpha_{1}\left(t_{1}\right)$ and $\alpha_{2}\left(t_{2}\right)$ at wavelengths $\lambda_{1}$ and $\lambda_{2}$, then category $c$ is not consistent with the observed spectral reflectance and $c$ is not a possible choice. If at a later observation time $t_{2}$, there is not a phenological growth stage of category $c$, which is a more mature growth stage than the earlier determined one at time $t_{1}$, and which yields spectral returns $\alpha_{1}\left(t_{2}\right)$ and $\alpha_{2}\left(t_{2}\right)$ at wavelengths $\lambda_{1}$ and $\lambda_{2}$, respectively, then category $c$ is not a possible choice. Note that spectral observations 
taken at a later calendar time are naturally constrained to be associated with more mature phenological growth stages than the phenological growth stages associated with earlier observation time.

\section{Bayesian Perspective}

In our Bayesian framework for multitemporal and multispectral classification of vegetation, we will assume that spectral reflectance is a function of vegetation category, vegetation growth state, and calendar time. Effects of atmospheric haze and geomorphologic soil and moisture variations and other kinds of catastrophies are neglected.

Let $G$ be the set of possible growth states. The growth states will depend on maturity, biomass, percent cover, and height of vegetation. We will assume that the growth stages of $G$ are ordered according to the natural maturing cycle which the vegetation undergoes. Let $\left\{t_{1}, \cdots, t_{N}\right\}$ be the set of observation times. The times in the set $T$ are naturally ordered by the relation earlier than or later than. Let $R$ be the set of possible reflectance values and $B=\{1,2, \cdots, M\}$ be the set of $M$ wavelengths bands of spectral reflectance that can be observed by the sensor.

Each spectral return vector $x$ is a member of the set $R^{M}$. The $m$ th component of $x$ is the spectral return using the $m$ th wavelength band of $B$. Suppose $x$ is a spectral reflectance vector of vegetation category $c$ in phenological growth stage $g$ at calendar time $t$. We denote the probability of observing $(x, c, g)$ at a given time $t$ by $P(x, c, g \mid t)$.

For multitemporal, multispectral data, the probability function of spectral reflectance vectors $x_{1}, \cdots, x_{N}$ coming from a small area ground patch of categories $c_{1}, \cdots, c_{N}$ in phenological growth stages $g_{1}, \cdots, g_{N}$ at calendar times $t_{1}, \cdots, t_{N}$, respectively, is denoted by

$$
P\left(x_{1}, \cdots, x_{N}, c_{1}, \cdots, c_{N}, g_{1}, \cdots, g_{N} \mid t_{1}, \cdots, t_{N}\right) .
$$

To determine a Bayes rule, the probability $P\left(x_{1}, \cdots, x_{N}\right.$, $\left.c_{1}, \cdots, c_{N} \mid t_{1}, \cdots, t_{N}\right)$ must be computed. Now,

$$
\begin{aligned}
P\left(c_{1}, \cdots, c_{N}, x_{1}, \cdots, x_{N} \mid t_{1}, \cdots, t_{N}\right) & \\
= & \sum_{g_{1}} \cdots \sum_{g_{N}} P\left(x_{1}, \cdots, x_{N}, c_{1}, \cdots, c_{N},\right. \\
& \cdot g_{1}, \cdots, g_{N} \mid\left(t_{1}, \cdots, t_{N}\right) \\
= & \sum_{g_{1}} \cdots \sum_{g_{N}} P\left(x_{1}, \cdots, x_{N} \mid c_{1}, \cdots, c_{N},\right. \\
& \left.\cdot g_{1}, \cdots, g_{N}, t_{1}, \cdots, t_{N}\right) \\
& \cdot P\left(c_{1}, \cdots, c_{N}, g_{1}, \cdots, g_{N} \mid t_{1}, \cdots, t_{N}\right) .
\end{aligned}
$$

We assume that the reflectance $x$ depends only on crop type $c$ and growth stage $g$ so that

$$
\begin{aligned}
& P\left(x_{1}, \cdots, x_{N} \mid c_{1}, \cdots, c_{N}, g_{1}, \cdots, g_{N}, t_{1}, \cdots, t_{N}\right) \\
& \quad=\prod_{n=1}^{N} P\left(x_{n} \mid c_{n}, g_{n}\right) .
\end{aligned}
$$

Likewise, we assume that vegetative growth is a Markov process depending on time and vegetation category alone so that

$$
\begin{gathered}
P\left(g_{1}, \cdots, g_{N} \mid c_{1}, \cdots, c_{N}, t_{1}, \cdots, t_{N}\right) \\
=\prod_{n=1}^{N} P\left(g_{n} \mid c_{n}, c_{n-1}, t_{n}, t_{n-1}\right)
\end{gathered}
$$

and

$$
\begin{aligned}
P\left(c_{1},\right. & \left.\cdots, c_{N}, g_{1}, \cdots, g_{N} \mid t_{1}, \cdots, t_{N}\right) \\
= & P\left(g_{1}, \cdots, g_{N} \mid c_{1}, \cdots, c_{N}, t_{1}, \cdots, t_{N}\right) \\
& \cdot P\left(c_{1}, \cdots, c_{N} \mid t_{1}, \cdots, t_{N}\right) \\
= & \prod_{n=1}^{N} P\left(g_{n} \mid c_{n}, c_{n-1}, g_{n-1}, t_{n-1}, t_{n}\right) \\
& \cdot P\left(c_{1}, \cdots, c_{N} \mid t_{1}, \cdots, t_{N}\right) .
\end{aligned}
$$

Hence, the probability for observed categories and multispectral reflectances is

$$
\begin{aligned}
P\left(c_{1}, \cdots, c_{N}, x_{1}, \cdots, x_{N} \mid t_{1}, \cdots, t_{N}\right) & =\sum_{g_{1}} P\left(x_{1} \mid c_{1}, g_{1}\right) P\left(g_{1} \mid c_{1}, t_{1}\right) \\
& \cdot \sum_{g_{2}} P\left(x_{2} \mid c_{2}, g_{2}\right) P\left(g_{2} \mid c_{1}, c_{2}, g_{1}, t_{1}, t_{2}\right) \\
& \cdots \cdot \sum_{g_{N}} P\left(x_{N} \mid c_{N}, g_{N}\right) \\
& \cdot P\left(g_{N} \mid c_{N-1}, c_{N}, g_{N-1}, t_{N-1}, t_{N}\right) \\
& \cdot P\left(c_{1}, \cdots, c_{N} \mid t_{1}, \cdots, t_{N}\right) .
\end{aligned}
$$

In theory, the formula just derived could be used to determine a Bayes rule in the usual way. In practice, there are too many distributions to estimate and too many calculations to do to calculate the required probabilities. However, because the required probability has the form of a product, if any probability in the product is zero, then the product must be zero. And a Bayes rule would never make an assignment to a category with a zero probability. This fact can be utilized to make an efficient table lookup rule which uses vegetation phenology just by storing in the table(s) those regions in measurement space having nonzero probability.

The astute reader will undoubtedly wonder why such a decision scheme has any chance of working at all. Why can't any spectral observation vector be possible for many growth stages for most categories? The reason that this is not possible is empirical. On all cases tested the probability distributions which were conditioned by crop growth state had resulting conditioned probability distributions with much smaller variance than the usual unconditioned ones. The conditioned ones are, therefore, much more peaked.

\section{Classification Using Phenological Vegetation Signatures and Prior Constraints}

Given observation times $t_{1}, \cdots, t_{N}$ during which a small area ground patch is observed, in the previous section we derived a formula for the probability of a small area ground patch having corresponding vegetation types $c_{1}, \cdots, c_{N}$ with 
respective spectral reflectance vectors $x_{1}, \cdots, x_{N}$. In this section we will show how this kind of representation for the probability can be used to define vegetation signatures and a classification method which can then be used to recognize vegetation type and vegetation growth state in a structural pattern recognition manner which is implementable as a table lookup rule.

For simplicity of discussion we will assume that for the observation times $t_{1}, \cdots, t_{N}$, the small area ground patch being observed does not change vegetation type and that the vegetation itself matures in a normal manner. We will also allow for the possible use of prior information which would indicate that at given observation times only certain growth states for the vegetation category are reasonable ones. Such prior constraints can come from historical crop calendar information, perhaps combined with a vegetation growth model that uses local weather temperature and moisture information.

If the vegetation category does not change over the period of observation so that $c_{i}=c$ for $i=1,2, \cdots, N$, then the probability derived in the last section is

$$
\text { (**) } \begin{aligned}
& P\left(x_{1}, \cdots, x_{N}, c \mid t_{1}, \cdots, t_{N}\right) \\
= & \left\{\prod _ { n = 1 } ^ { N } \left[\sum_{g_{n}} P\left(x_{n} \mid c, g_{n}\right)\right.\right. \\
& \left.\left.\cdot P\left(g_{n} \mid c, g_{n-1}, t_{n-1}, t_{n}\right)\right]\right\} \cdot P(c) .
\end{aligned}
$$

A necessary condition for a Bayes rule to assign the multitemporal, multispectral vectors $x_{1}, \cdots, x_{N}$ to category $c$ is for $P\left(x_{1}, \cdots, x_{N}, c \mid t_{1}, \cdots, t_{N}\right)$ to be nonzero. Since this joint probability is a product, if the joint probability is nonzero, then every term of the product must be greater than zero. This means that for each $n$ :

$$
\sum_{g_{n}} P\left(x_{n} \mid c, g_{n}\right) P\left(g_{n} \mid c, g_{n-1}, t_{n-1}, t_{n}\right)>0 \text {. }
$$

The product in each term of the above sum if nonzero iff $P\left(x_{n} \mid c, g_{n}\right)$ and $P\left(g_{n} \mid c, g_{n-1}, t_{n}, t_{n-1}\right)$ are both nonzero. The term $P\left(x_{n} \mid c, g_{n}\right)$ is nonzero iff the spectral reflectance vector $x_{n}$ is possible for vegetation category $c$ in growth state $g_{n}$. The term $P\left(g_{n} \mid c, g_{n-1}, t_{n-1}, t_{n}\right)$ is nonzero iff the crop calendar practices of the region being observed allow vegetation of category $c$ to be in growth state $g_{n}$ at time $t_{n}$ and the rate of growth of vegetation type $c$ is such that growth state $g_{n}$ can be reached from state $g_{n-1}$ in the time period from $t_{n-1}$ to $t_{n}$.

The set $S$ is called a signature for a category if it contains those data vectors whose components are spectral reflectances having nonzero probability for a growth state of a category. We can formalize a definition of $K$ th-order signature in a way that allows us to consider $K$-dimensional data vectors. Let $M$ be the number of wavelengths used. Let $x=\left(\alpha_{1}, \cdots, \alpha_{M}\right) \in R^{M}$, the set of possible observed reflectances, and denote by $\left(b_{1}, \cdots, b_{K}\right) \in B^{K}$ a selection of $K$ out of the $M$ bands. Let $B^{\prime} \subseteq B^{K}$. The $K$ th-order signature is defined with respect to only those $K$ tuples of bands in $B^{\prime}$. The Kth-order signatures of a category $c, S \subseteq G \times(R \times B)^{K}$, consists of all $(2 K+1)$ tuples of growth stage, band, reflectance, band, $\cdots$, reflectance, and band whose conditional probability is greater than zero.

$$
\begin{aligned}
S= & \left\{\left(g,\left(r_{1}, b_{1}\right), \cdots,\left(r_{K}, b_{K}\right)\right) \in G \times(R \times B)^{K} \mid\right. \\
& \text { for some }\left(b_{1}, \cdots, b_{K}\right) \in B^{\prime}, \\
& \left.P_{b_{1}}, \cdots, b_{K}\left(r_{1}, \cdots, r_{K} \mid g, c\right)>0\right\}
\end{aligned}
$$

where the pair $\left(r_{i}, b_{i}\right)$ denotes reflectance value corresponding band wavelength $b_{i}$ so that $r_{i}=\alpha_{b_{i}}$.

In an analogous way we can define a Kth-order observation relation $\Theta \subseteq T \times(R \times B)^{K}$ to consist of all those $(2 K+1)$ tuples of observation time, reflectance, band, $\cdots$, reflectance, and band which have been measured.

$$
\Theta=\left\{\left(t,\left(r_{1}, b_{1}\right), \cdots,\left(r_{K}, b_{K}\right) \in T \times(R \times B)^{K} \mid\right.\right.
$$

for some $\left(b_{1}, \cdots, b_{K}\right) \in B^{\prime}$ and for some $n$, $t=t_{n}$, the observed reflectance were $r_{i}$ on bands $b_{i}, \quad$ for $i=1,2, \cdots, K$ \}.

At each observation time at most one reflectance value is measured for each band. (There may be none if there is missing data). Note that the only $K$ tuples of reflectance bands used for observations are those $K$ tuples in $B^{\prime}$.

The set $C$ of prior constraints relating growth states to observation times and growth states at earlier times is

$$
C=\left\{\left(t_{1}, g_{1}, t_{0}, g_{0}\right) \in(T \times G)^{2} \mid P\left(g_{1} \mid t_{1}, t_{0}, g_{0}\right)>0\right\} \text {. }
$$

To determine if an observation $\Theta$ requires us to reject a vegetation category (that is, determine if $(* *)$ is nonzero) we will determine if for every $n 1$ ) there exists a growth state $g_{n}$ such that $P\left(x_{n} \mid c, g_{n}\right)$ is nonzero, and 2) if the $g_{n}$ is such that $P\left(g_{n} \mid c, g_{n-1}, t_{n-1}, t_{n}\right)$ is nonzero.

Let $H$ be a subset of $T \times G$ so that $H$ associates observation times with growth states. We define the relation composition (Haralick and Kartus, [14]) of $\Theta$ with $H$, written $\Theta \cdot H$ by

$$
\begin{aligned}
\Theta \cdot H= & \left\{\left(g,\left(r_{1}, b_{1}\right), \cdots,\left(r_{K}, b_{K}\right) \text { in } G \times(R \times B)^{K} \mid\right.\right. \\
& \text { for some } t \in T,\left(t,\left(r_{1}, b_{1}\right), \cdots,\left(r_{K}, b_{K}\right) \in \Theta\right. \\
& \text { and }(t, g) \in H\} .
\end{aligned}
$$

If $\Theta \cdot H$ is not a subset of $S$, then 1) is not satisfied. If $\Theta \cdot H$ is a subset of $S$ and $K=M$ and $H$ is defined everywhere on $T$, then 1) is satisfied. If $H \times H$ is a subset of $C$ and $H$ is defined everywhere on $T$, then 2 ) is satisfied.

Given $\Theta$ and $S$, let us find $H$ so that $\Theta \cdot H$ is a subset of $S$ and $H \times H$ is a subset of $C$ in the case where

$$
\begin{aligned}
C= & \left\{\left(t_{1}, g_{1}, t_{0}, g_{0}\right) \in(T \times G)^{2} \mid t_{1}>t_{0} \text { implies } g_{1}>g_{0}\right. \\
& \text { and } \left.\left(t_{1}, g_{1}\right) \text { and }\left(t_{0}, g_{0}\right) \text { in } C^{\prime}\right\}, \\
& \text { where } C^{\prime}=\{(g, t) \mid P(g \mid t)>0\} .
\end{aligned}
$$

This is the case where growth states are constrained to be chronologically ordered in time and consistent with observation times.

The table lookup implementation for finding $H$ is as follows. For each $K$ tuple $\left(b_{1}, \cdots, b_{K}\right)$ of bands in $B^{\prime}$ there is a table 
$T\left(b_{1}, \cdots, b_{K}\right)$, which gives lists of possible growth states for values of reflectances in bands $b_{1}, \cdots, b_{K}$. These tables together comprise the $K$ th-order signature $S$. There is a table $C^{\prime}$ listing possible growth states for a given observation times. The implementation works as follows.

Given $\Theta \subseteq T \times(R \times B)^{K}$, the existence of a function satisfying 1$)$ and 2$)$ is an easy matter to ascertain. Define $F\left(b_{1}\right.$, $\left.\cdots, b_{K}\right)$ by

$$
\begin{aligned}
F\left(b_{1}, \cdots, b_{K}\right)= & \left\{(t, g) \in C^{\prime} \mid\right. \\
& \text { for some }\left(r_{1}, \cdots, r_{K}\right) \text { in } R^{K}, \\
& \left.\left(t,\left(r_{1}, b_{1}\right), \cdots,\left(r_{K}, b_{K}\right)\right) \in S\right\} .
\end{aligned}
$$

Then with the properties of $\Theta$ already mentioned, the proposition at the end of this section states that any $H \subseteq C^{\prime}$ satisfying $\Theta \cdot H \subseteq S$ must be contained in

$$
\bigcap_{\left(b_{1}, \cdots, b_{K}\right) \in B^{\prime}} F\left(b_{1}, \cdots, b_{K}\right)
$$

and furthermore, the composition

$$
\Theta \cdot \bigcap_{\left(b_{1}, \cdots, b_{K}\right) \in B^{\prime}} F\left(b_{1}, \cdots, b_{K}\right)
$$

must be contained in $S$.

This implies that we can determine the existence of a function satisfying 1) and 2) by construction. First construct the relation $W$ in $C^{\prime}$ defined by

$$
W=\bigcap_{\left(b_{1}, \cdots, b_{K}\right) \in B^{\prime}} F\left(b_{1}, \cdots, b_{K}\right) .
$$

Then construct a monotonic part $H$ of $V$ in $W$. If this $H$ associates a growth state for each observation time then $H$ is a function satisfying 1) and 2).

For example, suppose $t_{1}$ is the first observation time. Using the table $C^{\prime}$ we retrieve a set of possible growth states $G_{1}$ and we check growth states in $G_{1}$ against observed reflectances until we find the earliest growth state consistent with the observed reflectances. We check a growth state in $G_{1}$ as follows. For each $K$ tuple of bands in $B^{\prime}$ we enter the corresponding observed reflectances at $t_{1}$ into the table $T\left(b_{1}, \cdots, b_{K}\right)$ and get back a set of growth states. If each such set contains the growth state we are considering, the growth state is consistent with the observed reflectances. At time $t_{2}$, we retrieve a set of possible growth states and intersect it with the set of possible growth states later than the earliest consistent growth state for $t_{1}$ to get $G_{2}$. Then find the earliest growth state in $G_{2}$ which is consistent with the observed reflectances at time $t_{2}, \cdots, \cdots$ and so on for each observation time.

\section{Proposition}

Let $\theta \subseteq T \times(R \times B)^{K}, S \subseteq G \times(R \times B)^{K}, H \subseteq C \subseteq T \times G$, and $B^{\prime} \subseteq B^{K}$. Define $F\left(b_{1}, \cdots, b_{K}\right)$ as follows:

$$
\begin{aligned}
F\left(b_{1}, \cdots, b_{K}\right)= & \{(t, g) \in C \mid \\
& \text { for some }\left(r_{1}, \cdots, r_{K}\right) \in R^{K}, \\
& \left(t, r_{1}, b_{1}, \cdots, r_{K}, b_{K}\right) \in \theta \\
& \text { and } \left.\left(g, r_{1}, b_{1}, \cdots, r_{K}, b_{K}\right) \in S\right\} .
\end{aligned}
$$

Suppose we have one set of measurements per observation for one small area of ground, so that for each $t \in T$ and $\left(b_{1}, \cdots\right.$, $\left.b_{K}\right) \in B^{\prime}$, there exists exactly one $\left(s_{1}, \cdots, s_{K}\right) \in R^{K}$ such that $\left(t, b_{1}, s_{1}, \cdots, b_{K}, s_{K}\right) \in \theta$. Then

$$
H \subseteq \bigcap_{\left(b_{1}, \cdots, b_{K}\right) \in B^{\prime}} F\left(b_{1}, \cdots, b_{K}\right)
$$

if and only if $\theta \cdot H \subseteq S$.

Proof: Suppose

$$
H \subseteq \bigcap_{\left(b_{1}, \cdots, b_{K}\right) \in B^{\prime}} F\left(b_{1}, \cdots, b_{K}\right) .
$$

Let $\left(g, r_{1}, \beta_{1}, \cdots, r_{K}, \beta_{K}\right) \in \theta \cdot H$. Then there exists a $t \in T$ such that $\left(t, r_{1}, \beta_{1}, \cdots, r_{K}, \beta_{K}\right) \in \theta$ and $(t, g) \in H$. Now $\left(t, r_{1}, \beta_{1}, \cdots, r_{K}, \beta_{K}\right) \in \theta$ implies $\left(\beta_{1}, \cdots, \beta_{K}\right) \in B^{\prime}$. Then $(t, g) \in H$ implies

$$
(t, g) \in \bigcap_{\left(b_{1}, \cdots, b_{K}\right) \in B^{\prime}} F\left(b_{1}, \cdots, b_{K}\right)
$$

thus $(t, g) \in F\left(\beta_{1}, \cdots, \beta_{K}\right)$.

Since $(t, g) \in F\left(\beta_{1}, \cdots, \beta_{K}\right)$, there exists some $\left(s_{1}, \cdots\right.$, $\left.s_{K}\right) \in R^{K}$ such that $\left(t, s_{1}, \beta_{1}, \cdots, s_{K}, \beta_{K}\right) \in \theta$ and $\left(g, s_{1}\right.$, $\left.\beta_{1}, \cdots, s_{K}, \beta_{K}\right) \in S$. But $\left(t, s_{1}, \beta_{1}, \cdots, s_{K}, \beta_{K}\right) \in \theta$ and $\left(t, r_{1}, \beta_{1}, \cdots, r_{K}, \beta_{K}\right) \in \theta$ implies $s_{i}=r_{i}, i=1, \cdots, K$. So we must have $\left(g, r_{1}, \beta_{1}, \cdots, r_{K}, \beta_{K}\right) \in S$ and $\theta \cdot H \subseteq S$.

Suppose $\theta \cdot H \subseteq S$. Let $(t, g) \in H \subseteq C$. Let $\left(\beta_{1}, \cdots, \beta_{K}\right) \in B^{\prime}$. Since there is one set of measurements for $t$, there exists $\left(s_{1}, \cdots, s_{K}\right) \in R^{K}$ so that $\left(t, s_{1}, \beta_{1}, \cdots, s_{K}, \beta_{K}\right) \in \theta$. Then $\left(t, s_{1}, \beta_{1}, \cdots, s_{K}, \beta_{K}\right) \in \theta$ and $(t, g) \in H$ implies $\left(g, s_{1}, \beta_{1}, \cdots\right.$, $\left.s_{K}, \beta_{K}\right) \in \theta \cdot H \subseteq S$. Now $(t, g) \in C,\left(t, s_{1}, \beta_{1}, \cdots, s_{K}, \beta_{K}\right) \in \theta$ and $\left(g, s_{1}, \beta_{1}, \cdots, s_{K}, \beta_{K}\right) \in S$ imply $(t, g) \in F\left(\beta_{1}, \cdots, \beta_{K}\right)$. Then

$$
H \subseteq \bigcap_{\left(\beta_{1}, \cdots, \beta_{K}\right) \in B^{\prime}} F\left(\beta_{1}, \cdots, \beta_{K}\right) .
$$

\section{TABle Lookup Rule Implementation}

(First-Order and No Prior Constraints)

In this section we specialize the implementation discussed in Section III for the first-order signature case with no prior constraints. A sufficient condition for $\Sigma_{g_{n}} p\left(x_{n} \mid c_{n}, g_{n}\right) p_{t_{n}}\left(g_{n} \mid c_{n}\right)$ to be zero is for $p\left(x_{n} \mid c_{n}, g_{n}\right)=0$ for all values of $g_{n}$. Let $x$ be a $K$-dimensional spectral reflectance observation. A sufficient condition for $p(x \mid c, g)=0$ for all growth values of $g$ is for there to be no phenological growth stage $g$ which gives a positive marginal conditional probability for each component $\alpha$ of the observed reflectance $x$. Let $P_{1} \cdots K_{K}\left(\alpha_{1}, \cdots, \alpha_{K} \mid c, g\right)$ be the probability of observing the $K$ spectral band reflectance $\left(\alpha_{1}, \cdots, \alpha_{K}\right)$ from a vegetation of type $c$ in growth state $g$. Let $P_{k}\left(\alpha_{k} \mid c, g\right)$ be the marginal probability of observing spectral reflectance $\alpha_{k}$ from band $k$ given vegetation type $c$ and growth stage $g$. Then a sufficient condition for $P_{1} \ldots K\left(\alpha_{1}, \cdots\right.$, $\left.\alpha_{K} \mid c, g,\right)=0$ is for $P_{k}\left(\alpha_{k} \mid c, g\right)=0$ for some spectral band $k$. If there is no phenological growth state which gives a positive marginal conditional probability for each component of the observed spectral reflectance $\left(\alpha_{1}, \cdots, \alpha_{K}\right)$, then

$$
\bigcap_{k=1}^{K}\left\{g \mid P_{k}\left(\alpha_{k} \mid c, g\right)>0\right\}=\phi .
$$


This leads to the following criteria for eliminating category assignments which a Bayes rule would also eliminate.

For a given $\epsilon \geqslant 0$, define the table by $R(k, \alpha, c)=\left\{g \mid P_{k}(\alpha \mid c\right.$, $g) \geqslant \epsilon\}$. Suppose multitemporal, multispectral returns of $\left(\alpha_{11}, \cdots, \alpha_{1 K}\right),\left(\alpha_{21}, \cdots, \alpha_{2 K}\right), \cdots,\left(\alpha_{N 1}, \cdots, \alpha_{N K}\right)$ are observed for calendar times $t_{1}, \cdots, t_{N}$. Then if

$$
\bigcap_{k=1}^{K} R\left(k, \alpha_{n k}, c\right) \neq \phi, \quad \text { for some } n
$$

a Bayes rule could not make the assignment to category $c$. If

$$
\bigcap_{k=1}^{K} R\left(k, \alpha_{n k}, c\right) \neq \phi, \quad \text { for all } n
$$

then category $c$ has not been eliminated and could be the category assigned by the Bayes rule. If

$$
\bigcap_{k=1}^{K} R\left(k, \alpha_{n k}, c^{*}\right) \neq \phi, \quad \text { for all } n
$$

and for every

$$
c \neq c^{*}, \bigcap_{k=1}^{K} R\left(k, \alpha_{n k}, c\right)=\phi, \quad \text { for some } n
$$

then a Bayes rule must make the assignment to the unique category $c^{*}$.

\section{EXAMPLES}

An example easily illustrates the first-order table lookup idea graphically. Fig. 1 shows graphs for the tables $R(k, \alpha, c)$. A square blacked in for coordinate $(g, \alpha)$ means that for the corresponding spectral value $\alpha$, the phenological growth stage $g$ belongs to the table $R$. Suppose that there are two spectral wavelengths band 1 and band 2, two categories, and two times at which observations are taken. Let the spectral observation for time 1 be $(9,10)$ and the spectral observation for time 2 be $(3,6)$. Examining the tables for category 1 , we have

$$
\begin{aligned}
R(1,9,1) & =\{3,5,6,7\} \\
R(2,10,1) & =\{0,1,2,3,17,18,19\} \\
R(1,9,1) \cap R(2,10,1) & =\{3\} .
\end{aligned}
$$

This means that the only time the observation $(9,10)$ could occur from category 1 is during phenological growth stage 3 . Examining the tables for category 2, we have

$$
\begin{aligned}
R(1,9,2) & =\{5,6,7,13,14\} \\
R(2,10,2) & =\{0,1,7,8,18,19\} \\
R(1,9,2) \cap R(2,10,7) & =\{7\} .
\end{aligned}
$$

This means that the only time the observation $(9,10)$ could occur from category 2 is during phenological growth stage 7 . So after the first spectral observation both categories are still possible.

Now consider the second observation $(3,6)$. By the tables

$$
\begin{aligned}
R(1,3,1) & =\{13,14\} \\
R(2,6,1) & =\{6,7,8,9,13,14\} \\
R(1,3,1) \cap R(2,6,1) & =\{13,14\} .
\end{aligned}
$$
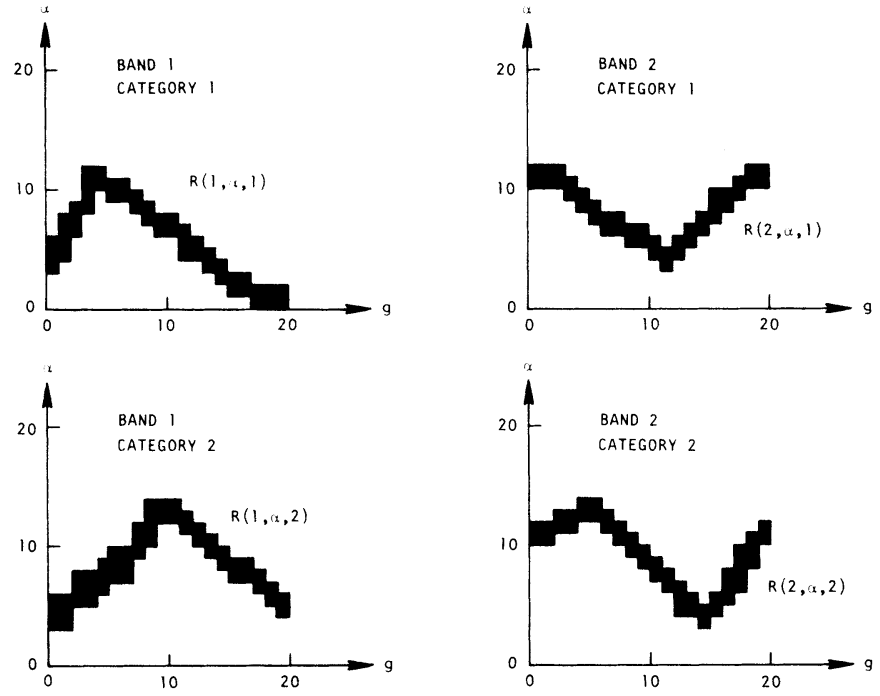

Fig. 1. Graphic illustration of the tables $R(b, \alpha, c)$. A square blacked in for coordinates $(g, \alpha)$ means that for the corresponding $\alpha$, the phenological growth stage $g \in R(b, \alpha, c)$ if and only if $P_{b}(\alpha \mid g, c)>$ $\epsilon \geqslant 0$ for some specified value of $G$.

This means that spectral observation $(3,6)$ is possible for category 1 only during phenological growth stages 13 and 14 .

By the tables

$$
\begin{aligned}
R(1,3,2) & =\{0,1\} \\
R(2,6,2) & =\{11,12\} \\
R(1,3,2) \cap R(2,6,2) & =\phi .
\end{aligned}
$$

This means that there is no phenological growth stage for category 2 which yields the spectral observation $(3,6)$. The conclusion, therefore, is that the small area ground patch having early spectral return of $(9,10)$ and later spectral return of $(3,6)$ must be an area of vegetation category 1 observed during its 3 and 13 or 14 phenological growth stages.

If instead of the intersection $R(1,3,2) \cap R(2,6,2)=\phi$, we had $R(1,3,2) \cap R(2,6,2)=\{4,6\}$, category 2 would be eliminated because the spectral reflectance it has at a late calendar time matches a possible spectral reflectance for category 2 only at early phenological growth states 4 or 6 , states before growth state 7 . Later calendar times must correspond to later phenological growth states.

\section{Empirical Determination of CATEgory Signatures}

In this section we suggest an estimation procedure to determine a category signature from a training data set. The procedure is iterative consisting of a step of dynamic programming minimization followed by averaging very much in the spirit of the ISODATA clustering technique [1].

Let $x(i, j, k)$ be the observed spectral reflectance from the $i$ th spectral band, $j$ th small area ground patch (or unit), taken at the $k$ th observation time. The set $\{x(i, j, k) \mid i=1, \cdots, I$; $j=1, \cdots, J ; k=1, \cdots, K\}$ is the training set for the category. Let $u$ be current mean spectral signature for the category of interest. $u(n ; i)$ is the mean $i$ th spectral band reflectance of a unit in the $n$th growth state for the category. The iterative 
procedure uses the training set and current mean and computes a new mean signature which is more representative of the data in the training set. The initial mean signature can be just one of the training vectors whose time components have been simply interpolated to growth states.

Before describing the procedure in detail, consider the meaning of $\delta=|x(i, j, k)-u(n ; i)|$. If the $k$ th observation time of the $j$ th unit corresponded to growth state $n$, then $\delta$ is the absolute value of the difference between the observed spectral reflectance of the $i$ th band and its mean. Define

$$
a(k, n ; j)=\max _{i}|x(i, j, k)-u(n ; i)| .
$$

The number $a(k, n ; j)$ is the maximum difference taken over all bands for the $j$ th unit when the $k$ th observation time corresponds to the $n$th growth state. In order to relate observation times to growth states in the best possible way, we must determine a strictly monotonic mapping $m$ which minimizes

$$
\sum_{k=1}^{K} a(k, m(j, k) ; j)
$$

Note that the sum of differences is taken over all observation times. We want the mapping $m$ to be monotonic so that later observation times are forced to be associated with later or more mature growth states. For each unit $j$, the mapping $m(j, k)$ can be determined by a dynamic programming optimization procedure [2].

Once the best mapping has been determined for each unit a new mean signature can be determined by averaging. Define the set $A_{n}$ as the set of all unit-observation time pairs which are mapped by $m$ to growth state $n$ :

$$
A_{n}=\{(j, k) \mid m(j, k)=n\} .
$$

Define the updated mean spectral signature $u^{\prime}$ by

$$
u^{\prime}(n, i)=\frac{\sum_{(j, k) \in A_{n}} r(i, j, k)}{\# A_{n}} .
$$

When the new updated spectral signature is different from the old spectral signature by an insignificant amount, the iterative procedure terminates. The final $u$ can be considered as the first-order signature skeleton of the category. This signature skeleton can be broadened to construct the signature. $\left(g, \alpha_{i}\right)$ is included in the signature if $\left|\alpha_{i}-u\left(g_{1} i\right)\right|<w$, where $w$ is the chosen signature width.

Once the first-order signature skeleton of a category has been defined, $K$ th-order signature skeleton can be defined in the following natural way. Let $B^{\prime} \subseteq B^{K}$ be a set of $K$ tuples of bands over which the $K$ th-order signature is to be defined. Let $A$ be the set of small area ground patches over which observations for the training data are made and let each observation consist of a reflectance value $r(t, a, b)$ which is a function of time $t$, small area ground patch $a$, and wavelength band $b$. The dynamic programming method begins with an observation $r=r(t, a, b)$ and first-order signature skeleton $u=u(g, b)$ and produces the best mapping $m=m(a, t)$ which associates each time $t$ and small area ground patch $a$ with the growth state $m(a, t)$. The $K$ th-order signature skeleton $S$ is just the set of reflectance values produced by the translation $m$ makes of time to growth states for the observations in the training data.

$$
S=\left\{\left(g, r_{1}, b_{1}, \cdots, r_{k}, b_{k}\right) \in G \times(R \times B)^{K} \mid\right.
$$

for some observation time $t$, small area ground patch $a$, and $K$ tuple of bands $\left(b_{1}, \cdots, b_{K}\right) \in B^{\prime}$, there exists an observation $r=r(t, a, b)$ in the training data with associated mapping $m=m(a, t)$ satisfying

1) $g=m(a, t)$, 2) $\left.r_{k}=r\left(t, a, b_{k}\right), k=1, \cdots, K\right\}$.

\section{IDENTIFICATION OF WhEAT IN MORTON County Using Phenological DiscRimination METHODS}

An extensive investigation of the use of phenological discrimination was carried out using Morton County Kansas LANDSAT from 5 observation dates in 1973-1974: October 23, 1973, May 9, 1974, May 27, 1974, June 14, 1974, and July 2, 1974. The image was 200 rows by 200 columns. The phenological discrimination procedure involves a number of choices for the user. The procedure involves two steps: 1) creation of the signature mean and 2) identification using the mean signature created in step 1). In this section we discuss the effects of the choices on the quality of classification as well as the validity of our use of the dynamic programming method for creation of mean signature.

\section{A. A Discussion of Results}

Consider the two steps in the discrimination procedure. In the first step the user chooses an input sample to train the signature and the number of growth states to be characterized in the signature. In the identification step the user chooses the "signature width" and which MSS band/observation date combinations to use. The choice of "signature width" is critical, especially when one is identifying only one crop class. The larger the "signature width" the larger the number of pixels that will be identified as in the crop class. The percent correct identification will increase with "width but at the cost of increased false identification. In the identification step the user also has the option of specifying a range of allowed growth states for each observation time. A good choice of these growth state restrictions effectively cuts down on the number of false classifications, without much reduction in the rate of correct classification.

Sample adequacy was investigated by comparing the discrimination results with no growth state restrictions using a random sample of 35 wheat field averages and several random samples of individual pixels. It seems that a random training sample of around 100 pixels (about 2.5 percent of the ground truth wheat) is of adequate size as discrimination was not significantly better with a sample of twice that size or with the field average samples. Our test set always consisted of the remaining 97.5 percent of our ground truths. 
We have performed 4 classifications of wheat with signatures having $5,10,20$, and 36 growth states, respectively. This is a range of 1 to 7 growth states per observation time, since we have 5 observations of the Morton County test site. The general shape of the mean signatures with differing numbers of growth states is the same. Our best discrimination was with a 36 growth state signature with a width of 3.25 . Using this signature and all observation dates, the results were 83-percent correct identification of ground truth wheat and 4-percent false identification. With a 5 growth state signature and a width of 6.0, the corresponding figures were 79 percent and 13 percent. The improved discrimination shows the usefulness of modeling several growth states per observation time.

The number of MSS bands needed for accurate identification was investigated. Most of our testing of the discrimination procedure has been done using MSS bands 4,5 , and 6 . However, it has been found that MSS bands 4 and 5 are sufficient for good wheat identification. Adding MSS band 7 reduced correct classifications significantly. Before testing it was thought that perhaps MSS bands 5 and 7 would be very useful for phenological discrimination of wheat, because they have often been most useful in other agricultural classification techniques. However, the identification of wheat with MSS bands 5 and 7 turned out not to be as good as with MSS bands 4 and 5 .

The possibility of accurate wheat identification with a single channel of information per observation time was investigated. Since, the phenological method of discrimination is a growth state identification process. It seemed likely that a single measure, indicating greenness of the pixel at the observation times, would be sufficient for identification of the crop. The four MSS band values for each observation data were transformed into Kauth greenness (Kauth and Thomas, [4]), a linear combination of the band values scaled to fit in the 0-31 integer value range.

$$
\begin{aligned}
K G= & 0.514(-0.290 M S S 4-0.562 M S S 5 \\
& +0.600 M S S 6+0.491 M S S 7)+13.6 .
\end{aligned}
$$

Wheat identification with this measure was not as good as identification with two or three MSS bands.

1) Prior Growth State Restrictions: Good wheat identification depends on the proper choice of growth state restrictions, especially if a subset of observation times are used. A description of a run using only two observation times will illustrate this. In this run growth states were restricted to states 1-5 for observation time 1 and states $10-12$ for observation time 2. The narrow choice of growth states allowed for the second observation time, May 9, is important because winter wheat is distinguished from other crop types principally because it is green on the May 9 date. Growth states 10-12 in the signature had low gray tone values in MSS band 5, which suggests by the Kauth greenness measure that they correspond to green states. Given the preceding growth state restrictions, 81 percent of the ground truth wheat was correctly identified and 5 percent of the nonwheat pixels were falsely labeled wheat.

The best choice of observation times was October 23 and May 9 for first-order discrimination of wheat. The best single observation time turned out to be May 9, as expected. The October 23 observation turned out to be the best addition to the May 9 observation. A third observation improved results significantly only when wheat was broken into two categories-quickly maturing wheat and slowly maturing wheat. The same 36 growth state signature was used to identify both subcategories of wheat, but with two sets of growth state restrictions. This discrimination resulted in a total of 83 percent of the wheat being identified, with only 4-percent false classification.

\section{B. Testing the Validity of Dynamic Programming in Mean Signature Generation}

Recall that different observation times may map into the same growth state in the construction of the mean signature. In order to test whether it is good to allow observations from different times to be used in the construction of growth state, an alternate procedure was tested. Let us say we have $G_{0}$ as the number of growth states per observation time. In each iteration we define a mapping $m:(j, t) \rightarrow G$ which minimizes

$$
\sum_{t=1}^{T} \max _{i}|x(i, j, t)-u(m(j, t) ; i)|
$$

for each sample $j$ with the additional restriction that the pair $(j, t)$ must map into one of the growth states in the set $\left\{(t-1) G_{0}+1,(t-1) G_{0}+2, \cdots, G_{0} t\right\}$. Because these sets are not overlapping, the method for finding the mapping turns out to be a simple minimization.

A few phenological discrimination runs using five observation dates were made using mean signatures generated by simple minimization. Discrimination was not quite as good as with similar runs using dynamic programming. The average standard deviation by band and growth state for the samples mapped into 20 growth states was higher with this simple minimization. This demonstrates the validity of combining observations with different dates in characterizing a signature growth state.

\section{Comparison of Phenological Discrimination with Other Procedures}

We identified wheat using Bayes table lookup and unsupervised clustering procedures developed at the University of Kansas Remote Sensing Laboratory and linear discrimination as implemented in the BMD package (Dixon, 1975). In our best phenological discrimination runs, using these procedures we achieved about 80-percent correct identification of wheat with about 5-percent false identification. The phenological method identified the wheat fields much better than unsupervised clustering, which had trouble separating wheat fields from summer fallow, probably because abandoned wheat fields were called summer fallow.

\section{CONCLUSION}

The growth state identification made in the discrimination process were the earliest growth states consistent with 1) the multispectral observations, 2) allowed growth states for observation date, and 3) the requirement that growth states be 
chronologically ordered. In order to use the growth state identification for information on crop maturity, it might be better to identify "best" consistent rather than earliest consistent growth states. Our identification may also be improved if our signature width varies with band and growth state. This idea led to limited testing of the use of "second-order" growth state signatures. These signatures account for covariance of spectral bands, as well as allowing signature width to vary with band and growth state. It is too early to tell if the secondorder signatures will lead to improved classification or give better information about crop maturity.

The phenological growth state procedure seems to be able to discriminate wheat about as well as some more standard procedures and label degree of maturity as well. Discrimination is comparable to discriminant analysis on Kansas wheat. The phenological method also identified corn well on a small site in Iowa.

\section{REFERENCES}

[1] G. H. Ball, "Data analysis in the social sciences: What about the details?" in Proc. Fall Joint Computer Conf., pp. 533-559, 1965.

[2] R. Bellman, Dynamic Programming. Princeton, N.J.: Princeton Univ. Press, 1957.

[3] K. S. Fu, D. A. Landgrebe, and T. L. Phillips, "Information processing of remotely sensed agricultural data," Proc. IEEE, vol. 57, pp. 639-653, 1969.

[4] R. J. Kauth and G. S. Thomas, "The tasselled cap-a graphic description of the spectral-temporal development of agricultural crops as seen by LANDSAT," in LARS Symp. Proc. Machine Processing of Remotely Sensed Data, Purdue Univ., West Lafayette, IN, (IEEE Cat. no. 76CH1103-1 MPRSD,) 1976.

[5] P. N. Misra and S. G. Wheeler, "Crop classification with LANDSAT multi-spectral scanner data," Pattern Recognition, vol. 10, pp. $1-13,1978$.

[6] S. G. Wheeler, P. N. Misra, and Q. A. Holmes, "Linear dimensionality of landsat agricultural data with implications for classification," in LARS Symp. Proc. Machine Processing of Remotely Sensed Data, Purdue Univ. Lafayette, IN, 1976.

\section{RELATED BIBLIOGRAPHY}

[7] G. H. Ball and D. J. Hall, "ISODATA, a novel method of data analysis and pattern classification," Stanford Research Inst., Menlo Park, CA, Apr. 1975.

[8] R. E. Carlson and C. Aspiazu, "Cropland acreage estimates from temporal, multi-spectral ERTS-1 data," Remote Sensing Environ., vol. 4, pp. 237-243, 1975.

[9] J. L. Engvall, J. D. Tubbs, and Q. A. Holmes, "Pattern recognition of LANDSAT data based upon temporal trend analysis," Remote Sensing Environ., vol. 6, pp. 303-314, 1977.

[10] L. D. Erickson and R. F. Nalepka, "PROGRAMS: A second generation multi-spectral, multi-temporal processing system for agricultural mensuration," in LARS Symp. Proc. Machine Pro- cessing of Remotely Sensed Data, Purdue Univ., West Lafayette, IN, (IEEE Cat. no. 76CH1103-1 MPRSD), 1976.

[11] P. T. Gammon, D. Malone, P. D. Brooke, and V. Carter, "Three approaches to the classification and mapping of inland wetlands," in Proc. Eleventh Symp. Remote Sensing of Environment, Univ. of Michigan, Ann Arbor, 1977.

[12] P. T. Gammon and V. P. Carter, "Comparison of vegetation classes in the great dismal swamp using two individual LANDSAT images and a temporal composite," in LARS Symp. Machine Processing of Remotely Sensed Data, Purdue Univ., West Lafayette, IN, (IEEE Cat. no. CH1103-IMPRSD), 1976.

[13] R. M. Haralick, "The table look-up rule," Commun. Statis. Theor. Math. A5, vol. 12, pp. 1163-1191, 1976.

[14] R. M. Haralick and J. Kartus, "Arrangements and homomorphisms," IEEE Trans. Syst., Man, Cybern., vol. SMC-8, p. 600612, Aug. 1978.

[15] T. Kaneko, "Crop classification using time features computed from multi-temporal multi-spectral data," presented at the 1978 Pattern Recognition Conf., Japan, 1978.

[16] Z. Kalensky and L. R. Scherk, "Accuracy of forest mapping from LANDSAT computer compatible tapes," presented at the Tenth Int. Symp. Remote Sensing of Environment, Ann Arbor, MI, Oct. 6-10, 1975

[17] E. T. Kanemasu, "Seasonal canopy reflectance patterns of wheat, sorghum, and soybeans," Remote Sensing Environ., vol. 3, pp. 43-47, 1974.

[18] R. J. Kauth, A. P. Pentand, and G. S. Thomas, "BLOB: An unsupervised clustering approach to spatial preprocessing of MSS imagery," presented at the Eleventh Int. Symp. Remote Sensing of Environment, Univ. of Michigan, Ann Arbor, 1977.

[19] R. J. Kauth and G. S. Thomas, "The tasselled cap-a graphic description of the spectral-temporal development of agricultural crops as seen by LANDSAT," in LARS Symp. Proc. Machine Processing of Remotely Sensed Data, Purdue Univ., West Lafayette, IN, (IEEE Cat. no. 76CH1103-1 MPRSD), 1976.

[20] D. A. Landgrebe et al., "A study of the utilization of ERTS-1 data from the Wabash River Basin," Purdue Univ., West Lafayette, IN, LARS, NASA Contract NAS 5-21773, Final Rep., 1974.

[21] T. Y. Le Toan, C. Cassirame, and J. Quash, "Inventory of rice fields in France using LANDSAT and aircraft data," in Proc. Eleventh Symp. Remote Sensing of Environment, Univ. of Michigan, Ann Arbor, 1977.

[22] J. Megier, "Multi-temporal digital analysis of LANDSAT data for inventory of poplar planted groves in north Italy," in Proc. Int. Symp. on Image Processing, Interactions with Photogrammetry and Remote Sensing, Graz, Oct. 3-5, 1977.

[23] P. N. Misra and S. G. Wheeler, "Crop classification with LANDSAT multi-spectral scanner data," Pattern Recognition, vol. 10, 1978, pp. 1-13.

[24] R. F. Nalepka, J. E. Colwell, and D. P. Rice, "Forecasts of winter wheat yield and production using LANDSAT data," Univ. of Michigan, Ann Arbor, NASA Contract NAS5-22398, Final Rep., Dec. 1977.

[25] Salmon-Drexler, "Reducing LANDSAT data to parameters with physical significance and signature extension," in Proc. Eleventh Int. Symp. Remote Sensing of Environment, Univ. of Michigan, Ann Arbor, 1977.

[26] Von Steen, D. A. and W. H. Wigton, "Crop identification and acreage measurement utilizing LANDSAT imagery," Statistical Reporting Service, US Dept. of Agriculture, Mar. 1976. 